\title{
Visceral Leishmaniasis in Children in Southern Turkey: Evaluation of Clinical and Laboratory Findings and Liposomal Amphotericin B Treatment
}

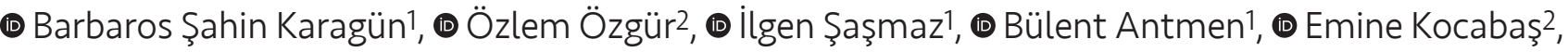 \\ (1) Emre Alhan² \\ ${ }^{1}$ Çukurova University Faculty of Medicine, Department of Pediatric Hematology and Oncology, Adana, Turkey \\ ${ }^{2}$ Çukurova University Faculty of Medicine, Department of Pediatric Infection Disease, Adana, Turkey
}

\begin{abstract}
Aim: Visceral leishmaniasis (VL) is a systemic infection that spreads hematogenously and affects the reticuloendothelial system by the infection of macrophages. VL occurs commonly in children, and only rarely in adults. VL should be considered in patients with prolonged high fever, hepatosplenomegaly, pancytopenia, weight loss, pallor and hypergammaglobulinemia.

Materials and Methods: In this study, a total of 18 pediatric patients -9 (50\%) males and 9 (50\%) females- treated for VL at our clinic from January 2004 to July 2014 were analyzed retrospectively. Average time from symptom onset to hospital admission was 64 \pm 21 days (range: 30-100 days). The mean age of patients was $88 \pm 40$ months (range: 36-182 months).

Results: The most common symptom at presentation was fever (88.9\%). Other common symptoms were fatigue, chills, weight loss and anorexia. Physical examination revealed splenomegaly and hepatomegaly in all patients. Anemia (92.4\%), leukopenia (78.7\%) and thrombocytopenia (76.2\%) were the most prominent laboratory abnormalities and $82.2 \%$ of the patients were pancytopenic on admission. Bone marrow smear was positive for leishmania in $100 \%$ of the patients. All patients received treatment with liposomal amphotericin B.

Conclusion: In certain regions, increased humidity rates associated with construction of dams and irrigation canals may lead to changes in the ecological balance and thus cause an increase in the population of disease-spreading vectors. Additionally, recent migration from the middle-eastern region to western parts of the world due to regional civil wars may have contributed to the observed increase in the incidence of various diseases such as VL.
\end{abstract}

Keywords: Leishmania, visceral leishmaniasis, liposomal Amphotericin B, child, Turkey

\section{Introduction}

Leishmaniasis is a form of parasitic disease with zoonotic-anthroponotic features which is caused by protozoa belonging to the kinetoplastida order, which are transmitted by the bite of sand flies (Phlebotomus or Lutzomyia) (1). It is considered as an epidemic disease which manifests with severe systemic effects. The disease may present as cutaneous, mucocutaneous or visceral leishmaniasis (VL). Globally, around 500.000 new VL cases are reported annually with a death toll of 60.000 (2). In this respect, it is accepted as the second most common cause of mortality among all tropical diseases (3). Vector borne diseases are an important public health problem in Turkey, especially in humid regions and regions that have borders 
with neighboring war-ridden countries in which public health-care is on the verge of collapse. Officially, there have been $207 \mathrm{VL}$ cases in Turkey during the last decade. The ongoing refugee crisis and the favorable climate of these regions may be contributing to the spread of vector-borne diseases (4).

$\mathrm{VL}$ is a systemic infection in which macrophages are infected through hematogenous spread. In developed western countries, a patient with $\mathrm{VL}$ is a rare occurrence and it is seen as an opportunistic infection affecting those with immunosuppression. In the tropical climate zone and Mediterranean basin countries, it occurs endemically (5). The most common etiologic agents include Leishmania (L) donovani, L. infantum and L. chagasi, while L. tropica and L. amazonensis are also known to cause the disease. In Turkey, it is seen endemically in the Southeastern, Mediterranean and Aegean regions. Clinically, it is characterized by a sustained high fever that is resistant to non-specific treatments and which has the following symptoms: pallor, weight loss, hepatosplenomegaly, pancytopenia and hypergammaglobulinemia. Due to these clinical and laboratory features, it resembles malaria, tuberculosis, brucellosis, salmonella infections or malign hematologic diseases (1-3). Thus, it is important to remind clinicians of the characteristics of $\mathrm{VL}$ in order to prevent misdiagnoses and provide prompt treatment to patients, especially considering the rarity of $\mathrm{VL}$ cases.

Our aim was to review the relevant literature and to investigate the characteristics of pediatric $\mathrm{VL}$, which is occasionally endemic in our region and presents a serious health problem in our country.

\section{Materials and Methods}

In this study, 18 pediatric patients diagnosed and treated for VL in our clinic between January 2004 and July 2014 were evaluated retrospectively. Demographic characteristics and the clinical and laboratory findings of all cases were retrospectively assessed from their clinical records.

All patients underwent bone marrow aspiration (BMA) for diagnostic purposes. BMA samples were assessed by the departments of Pediatric Hematology and Pediatric Infectious Diseases. The diagnoses of all patients were confirmed by the demonstration of parasite amastigotes in Giemsa-stained smears prepared from BMA samples. In five patients $(27.8 \%)$, promastigotes were isolated from cultures in Novy-MacNeal-Nicole (NNN) medium (Figure 1). Additionally, the RK-39 dipstick test was performed with all bone marrow aspirates.

In order to confirm the etiology of non-specific fever and hepatosplenomegaly, all patients were examined with posteroanterior chest $\mathrm{X}$-ray, purified protein derivative test, blood-, urine-, throat- and sputum cultures, thick blood smear, peripheral blood smear, serum Wright agglutination test, Gruber-Widal agglutination test, echocardiography, toxoplasmosis immunoglobulin M (IgM), CMV IgM, EBV VCA-IgM and collagen tissue disorder markers (antinuclear antibody, Anti-DNA, RF) for differential diagnosis of VL.

All patients underwent BMA; the aspiration was repeated in 4 patients with non-specific results to confirm the diagnosis. Three cases were diagnosed by the incubation of promastigotes in NNN media culture. Bone marrow or liver biopsies and spleen aspirations were not required in any of the patients.

All patients were treated with liposomal Amphotericin $B$ (Ambisome) administered $3 \mathrm{mg} / \mathrm{kg} /$ day parenterally on days 1-5, 14 and 21 (a total of 7 doses). Post-treatment cure was determined as diminishment of symptoms, normal body temperature, regression of spleen size, normalization of laboratory findings and finally, the absence of leishmania amastigotes on repeat bone marrow aspirates. Because of the retrospective nature of this study, it is outside the scope of the ethics committee, and so we did not apply to ethics committee. The parents of the patients included in this study provided informed consent for the patients data to be used.

\section{Results}

The study population included $18 \mathrm{VL}$ patients, 9 of whom were male (50\%) and 9 were female (50\%). The mean age of the patients was $88 \pm 40$ (range: $36-182$ ) months. The provinces where the patients resided were Adana (5), Hatay (4), Şanlıurfa (3), Osmaniye (2), Diyarbakır (2), Gaziantep (1) and Kahramanmaraş (1); all of these cities are located in Southern Turkey and have relatively humid climates due to their proximity to the Mediterranean Sea. Eleven patients $(61.1 \%)$ were living in a rural setting, while 7 (38.9\%) were from urban areas. Twelve patients $(66.7 \%)$ had a history of contact with animals. None of the patients had a previously diagnosed immunosuppressive condition. All patients had

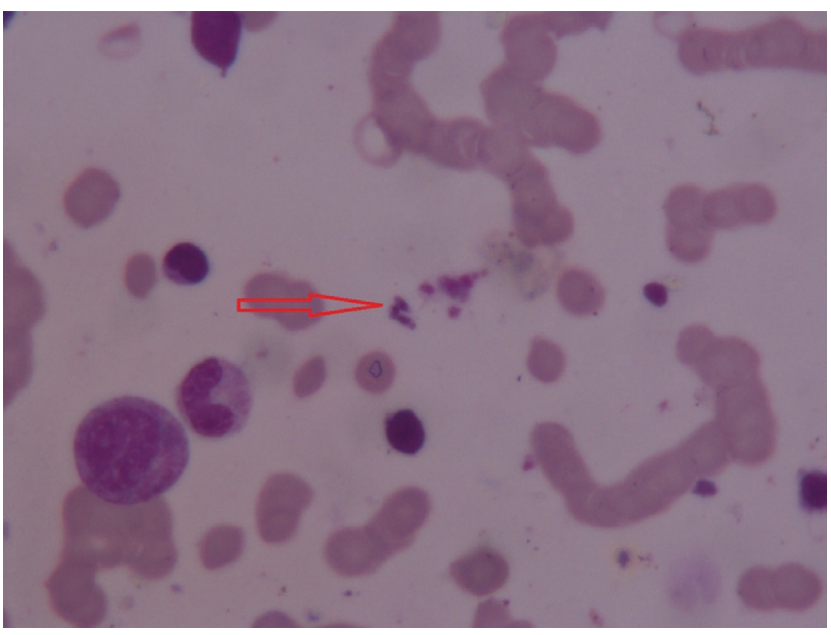

Figure 1. Light-microscopic examination of a stained bone marrow 
received inpatient antibiotic treatment in another hospital before admission to our clinic.

The most common complaint on admission was fever $(88.9 \%)$ (Table I). Other common symptoms were fatigue, chills, weight loss and loss of appetite. Physical examination revealed hepatomegaly and splenomegaly in all patients. Time from onset of symptoms to diagnosis was $64.05 \pm 21.37$ days (30-100 days). Laboratory test results revealed pancytopenia, hypoalbuminemia and a high erythrocyte sedimentation rate (ESR) (Table II).

\section{Discussion}

Leishmaniasis is a zoonotic infection which is endemic in the Mediterranean and Aegean regions of Turkey, while the south-eastern region has seen an increase in the number

\begin{tabular}{|l|l|l|}
\hline \multicolumn{3}{|l|}{ Table I. Frequency of symptoms for visceral leishmaniasis on } \\
admission
\end{tabular}

Table II. Laboratory and physical examination findings of visceral leishmaniasis

\begin{tabular}{|l|l|l|}
\hline Sign & No & \% \\
\hline Anemia & 18 & 100 \\
\hline Thrombocytopenia & 18 & 100 \\
\hline Neutropenia & 18 & 100 \\
\hline Splenomegaly & 18 & 100 \\
\hline Hepatomegaly & 18 & 100 \\
\hline Elevation of liver enzymes & 18 & 100 \\
\hline Increased ESR and CRP & 18 & 100 \\
\hline Pyrexia & 15 & 83.3 \\
\hline Lymphadenopathy & 9 & 50 \\
\hline Hypoalbuminemia & 6 & 33.3 \\
\hline Cachexia & 4 & 22.2 \\
\hline Petechia & 2 & 11.1 \\
\hline
\end{tabular}

ESR: Erythrocyte sedimentation rate, CRP: C-reactive protein of cases reported in the last few years. Official reports put the total number of VL cases during the last decade at 207. We hereby report 18 cases with VL that were diagnosed and treated at our center. None of the patients had a recurrence during 6 months of follow up.

\section{Initial Symptomatology}

Sixteen $(88.9 \%)$ of our patients presented with fever, while fatigue $(77.8 \%)$ and chills $(77.8 \%)$ were the second most common symptoms at presentation. Other symptoms in order of reducing frequency were as follows: loss of appetite $(66.7 \%)$, pallor $(55.6 \%)$, weight loss $(55.6 \%)$, abdominal distension (33.2\%), low blood count (22.2\%), cough (22.2\%), and diarrhea (16.7\%) (Table I). Similar to our findings, fever has been reported tof be the most frequent symptom among VL patients. Fever frequency has been reported as $94.2 \%$ in a study from Iran (6), 100\% in the Black Sea Region of Turkey (7), 95\% in Greece (8), 95\% in France (9), and $98 \%$ in China (10). Although the literature on $\mathrm{VL}$ is rather unanimous regarding symptom frequency, a few studies which report varying findings exist. For instance, a study by Krepis et al. (11) reported that all $(100 \%)$ of their patients had pallor; however, in the current study, pallor was observed in only $55.6 \%$ of patients, while Miao et al. (10) reported a frequency of $33 \%$. Among our patients, loss of appetite (66.7\%) and weight loss (55.6\%) were also primary symptoms, while in the study by Krepis et al. (11) loss of appetite and subsequent weight loss were evaluated together, and were observed in just $32.6 \%$ of their cases. The same study reported that $16.3 \%$ of their cases had vomiting/diarrhea, which is very similar to our findings.

\section{Physical Examination and Laboratory Findings}

All patients were found to have hepatosplenomegaly at presentation (100\%), while pyrexia was found in $83.3 \%$, lymphadenopathy in $50 \%$, cachexia in $22.2 \%$, and petechiae in $11.1 \%$. In other studies, splenomegaly (86-100\%) and hepatomegaly (58.1-98\%) are also reported to be the most frequent physical examination findings (6,10-12) (Table II). However, the frequency of hepatomegaly $(100 \%)$ in the current study group was relatively high compared to studies by Krepis et al. (11) (58.1\%), Abdinia et al. (6) (51.3\%) and Miao et al. (10) (74\%). It is also important to note that Abdinia et al. (6) found that liver involvement was present in all cases of mortality in their patient group. Lymphadenopathy was the third most common finding in our study group with a frequency of $50 \%$, however, Miao et al. (10) reported that it was present in only $33 \%$ of their patients. In the current study, bleeding manifestations were observed in the form of petechiae in $2(11.1 \%)$ patients, Krepis et al. (11) reported bleeding manifestations in $7 \%$ of their patients (12), while Abdinia et al. (6) reported petechiae and purpura in $11.5 \%$ of their study group. 
The classic triad of VL is comprised of fever, splenomegaly and pallor $(13,14)$. In the current study, the frequency of fever and splenomegaly conformed to the triad; however, pallor was observed in only $55.6 \%$ of patients. This is an irregular finding which may be caused by several of the differences between study populations including race, time until admission to center and previous treatments at other centers.

Laboratory findings in VL comprise varying levels of anemia (normocytic and normochromic), neutropenia, eosinopenia, thrombocytopenia, and an elevation of bilirubin and liver enzymes (15). In the current study, significant anemia, leukopenia, increased ESR and C-reactive protein (CRP) in addition to slight-to-moderate thrombocytopenia were present in all cases. Also, the majority of patients had decreased albumin levels with normal total protein concentration. Similarly, Krepis et al. (11) reported a decrease in the albumin/globulin ratio in $72.1 \%$ of their patients.

Anemia, slight-to-moderate liver enzyme elevation, and increased ESR and CRP were present in all (100\%) of our patients at presentation, which conforms to the majority of previous studies $(1,5,13)$. However, in a study by Abdinia et al. (6), aspartate aminotransferase (AST) and alanine aminotransferase (ALT) elevation were reported in only $25 \%$ and $11 \%$ of patients, respectively. This can be seen as a major difference, especially considering that the study in question was performed in a region that neighbors the south-eastern region of Turkey (Northwest of Iran). The majority of patients in our study resided in areas close to the Mediterranean Sea; whereas the Northeast of Iran is close to the Caspian Sea. Therefore, there may have been major differences in the type of Leishmania and/or vectors in these regions. Another cause for this difference may be simply explained by differences in reference ranges for AST and ALT in various studies.

\section{Treatment}

Leishmaniasis is traditionally treated with antimonial drugs, namely sodium stibogluconate and meglumine antimoniate (16). Although these are effective and cheap drugs, antimonials may cause severe cardiac and gastrointestinal side-effects (17). Miltefosine is another widely used treatment option with less frequent and less severe side effects compared to antimonials. However, a particular drawback of miltefosine is its lesser efficacy in those with human immunodeficiency virus, which could limit its use in developed countries where VL usually develops as an opportunistic infection in those with immunosuppression (18). Liposomal Amphotericin B is currently considered the most effective and safe drug for the treatment of $V L$ and the only drug with FDA approval (19), even though it is very expensive compared to the aforementioned treatments.
All of our patients received treatment with liposomal Amphotericin B at a parenteral dose of $3 \mathrm{mg} / \mathrm{kg}$ applied on days 1 to 5, 14 and 21 (cumulative dose of $21 \mathrm{mg} / \mathrm{kg}$ ). All patients were cured, no side effects were observed and none of the patients had a relapse during 6 months of follow-up.

The dosage used in the current study was relatively high compared to suggestions in studies from India that describe $89 \%$ cure rate with a cumulative dose as low as $3.75 \mathrm{mg} / \mathrm{kg}$ and $97 \%$ with $15 \mathrm{mg} / \mathrm{kg}$ (20). However, studies from other regions report lower cure rates with such doses. For instance, in Brazil, a treatment dose of $2 \mathrm{mg} / \mathrm{kg}$ per day for 10 days (total $20 \mathrm{mg} / \mathrm{kg}$ ) was found to cure $87 \%$ of patients, while a total dose ranging between 7-14 mg/ $\mathrm{kg}$ was successful in only $62 \%$ of cases. Various studies from different parts of the world have confirmed that a cumulative dose of $18-21 \mathrm{mg} / \mathrm{kg}$ achieves almost complete success in patients with VL (21-23). As such, the current FDA recommendation for the treatment of $V L$ with liposomal amphotericin B is $3 \mathrm{mg} / \mathrm{kg}$ on days 1-5, 14, and 21 (cumulative dose: $21 \mathrm{mg} / \mathrm{kg}$ ) (19). It is also important to note that some studies suggest that a high initial dosage $(5-10 \mathrm{mg} / \mathrm{kg})$ is instrumental in obtaining optimal tissue levels as soon as possible (24). This approach may also reduce hospital stay and is reported to be preferred by some pediatricians in Europe (12).

\section{Conclusion}

While our patient group showed significant differences in some clinical and laboratory findings compared to previous studies, the $100 \%$ cure rate of VL with a total dose of 21 $\mathrm{mg} / \mathrm{kg}$ is a demonstration of the efficacy of treatment with liposomal Amphotericin B. Recommendations of short- and long-term regimens are still a matter of debate, as both treatment courses have been shown to be effective in various studies. However, it is crucial to evaluate regional differences when considering treatment approach and we suggest following the FDA-approved treatment guideline in the majority of cases; however, severe cases may benefit from an initial high dosage. Further studies with varying dosage evaluations that are prospectively designed should be performed in order to elucidate optimal treatment with liposomal amphotericin B.

\section{Ethics}

Ethics Committee Approval: Retrospective study.

Informed Consent: The parents of the patients included in this study provided informed consent for the patients data to be used.

Peer-review: External and internal peer-reviewed.

\section{Authorship Contributions}

Surgical and Medical Practices: B.Ş.K., E.K., E.A., I.Ş., B.A., Concept: B.Ş.K., Ö.Ö., Design: B.Ş.K., Ö.Ö., E.A., Data Collection or Processing: B.Ş.K., Ö.Ö., I.Ş., Analysis or 
Interpretation: B.Ş.K., E.K., İ.Ş., B.A., Literature Search: B.Ş.K., E.K., I.Ş., Writing: B.Ş.K.

Conflict of Interest: No conflict of interest was declared by the authors.

Financial Disclosure: The authors declared that this study has received no financial support.

\section{References}

1. Kurşun E, Turunç T, Demiroğlu YZ, Solmaz S, Arslan H. Evaluation of fourteen adult cases with visceral leishmaniasis. Mikrobiyol Bul 2013;47:500-6.

2. Ozensoy S, Ozbel $\mathrm{Y}$, Turgay $\mathrm{N}$, et al. Serodiagnosis and epidemiology of visceral leishmaniasis in Turkey. Am J Trop Med Hyg 1998;59:363-9.

3. Mathers CD, Ezzati M, Lopez AD. Measuring the burden of neglected tropical diseases: the global burden of disease framework. PLoS Negl Trop Dise 2007;1:e114.

4. Gürel MS, YesilovaY, Ölgen MK, Özbel Y. Cutaneous leishmaniasis in Turkey. Türkiye Parazitolojii Dergisi 2012;36:121.

5. Karimkhani C, Wanga V, Coffeng LE, Naghavi P, Dellavalle RP, Naghavi M. Global burden of cutaneous leishmaniasis: a crosssectional analysis from the Global Burden of Disease Study 2013. Lancet Infect Dis 2016;16:584-91.

6. Abdinia B, Oliaei-Motlagh M, Teimouri-Dereshki A. Pediatric visceral leishmaniasis in northwest of Iran. Medicine (Baltimore) 2016;95:e5261.

7. Totan M, Dagdemir A, Muslu A, Albayrak D. Visceral childhood leishmaniasis in Turkey. Acta paediatr 2002;91:62-4.

8. Maltezou HC, Siafas C, Mavrikou M, et al. Visceral leishmaniasis during childhood in southern Greece. Clin Infect Dis 2000;31:113943.

9. Minodier P, Garnier J. Childhood visceral leishmaniasis in Provence. Arch Pediatr 2000;7:572-7.

10. Miao R, Wang Z, Guo Q, et al. Clinical and epidemiologic features of visceral leishmaniasis in children in southwestern china: A retrospective analysis from 2001 to 2015. Pediatr Infect Dis ) 2017;36:9-12.

11. Krepis P, Krepi A, Argyri l, et al. Childhood visceral leishmaniasis: distinctive features and diagnosis of a re-emerging disease. An 11-year experience from a tertiary referral center in Athens, Greece. Pediatr Infect Dis | 2018;37:419-23.
12. Kafetzis DA, Velissariou IM, Stabouli S, Mavrikou M, Delis D, Liapi C. Treatment of paediatric visceral leishmaniasis: amphotericin B or pentavalent antimony compounds? Int I Antimicrob Agents 2005;25:26-30.

13. Kafetzis DA. An overview of paediatric leishmaniasis. J Postgrade Med 2003;49:31-8.

14. Hamid GA, Gobah GA. Clinical and hematological manifestations of visceral leishmaniasis in Yemeni children. Turk J Hematol 2009;26:25-8.

15. Guerrant RL, Walker DH, Weller PF. Tropical infectious diseases: principles, pathogens and practice (Expert Consult--Online and Print), 3e: Saunders; 2011.

16. Jha TK, Olliaro P, Thakur CP, et al. Randomised controlled trial of aminosidine (paromomycin) $v$ sodium stibogluconate for treating visceral leishmaniasis in North Bihar, India. BMJ 1998;316:1200-5.

17. Chulay JD, Fleckenstein L, Smith DH. Pharmacokinetics of antimony during treatment of visceral leishmaniasis with sodium stibogluconate or meglumine antimoniate. Trans R Soc Trop Med Hyg 1988;82:69-72.

18. Moore EM, Lockwood DN. Treatment of visceral leishmaniasis. J Glob Infect Dis 2010;2:151-8.

19. Meyerhoff A. U.S. Food and drug administration approval of AmBisome (liposomal amphotericin B) for treatment of visceral leishmaniasis. Clin Infect Dis1999;28:42-8.

20. Sundar S, Jha TK, Thakur CP, Mishra M, Singh VR, Buffels R. Low-dose liposomal amphotericin $B$ in refractory Indian visceral leishmaniasis: a multicenter study. Am I Trop Med Hyg 2002;66:143-6.

21. Seaman J, Boer C, Wilkinson R, et al. Liposomal amphotericin B (AmBisome) in the treatment of complicated kala-azar under field conditions. Clin Infect Dis 1995;21:188-93.

22. Davidson RN, Di Martino L, Gradoni L, et al. Liposomal amphotericin B (AmBisome) in Mediterranean visceral leishmaniasis: a multi-centre trial. Q I Med 1994;87:75-81.

23. Syriopoulou V, Daikos GL, Theodoridou M, et al. Two doses of a lipid formulation of amphotericin B for the treatment of Mediterranean visceral leishmaniasis. Clin Infect Dis 2003;36:560-6.

24. Adler-Moore ), Proffitt RT. AmBisome: liposomal formulation, structure, mechanism of action and pre-clinical experience. $)$ Antimicrob Chemother 2002;49:21-30. 